\title{
Electrotriggered, Spatioselective, Quantitative Gene Delivery into a Single Cell Nucleus by Au Nanowire Nanoinjector
}

Seung Min Yoo, ${ }^{\ddagger \perp}$ Mijeong Kang, ${ }^{\dagger, \perp}$ Taejoon Kang, ${ }^{\S}$ Dong Min Kim,, Sang Yup Lee, ${ }^{*}$, and Bongsoo Kim*,†

\author{
${ }^{\dagger}$ Department of Chemistry and ${ }^{\star}$ Department of Chemical and Biomolecular Engineering (BK21 Program), KAIST, Daejeon 305-701, \\ Korea \\ ${ }^{\S}$ BioNanotechnology Research Center, KRIBB, Daejeon 305-806, Korea \\ "Department of Medical System Engineering, GIST, Gwangju, 500-712, Korea
}

Supporting Information

ABSTRACT: Delivery of bioactive materials into a cell is highly important in the study of cell biology and medical treatments. Ideal nanoinjectors should be able to deliver biomaterials with high spatial resolution while causing minimum cell damage. We developed a Au nanowire (NW) nanoinjector that has the thinnest diameter $(100-150 \mathrm{~nm})$ among the DNA delivering devices as well as optimum mechanical properties, minimizing cell damage. Well-defined (111) single-crystalline $\mathrm{Au}$ surface and high electric conductivity of a $\mathrm{Au} \mathrm{NW}$ nanoinjector allow precisely timed and efficient electrochemical release of DNA molecules attached on a Au NW surface. Both linear DNA and plasmid DNA were delivered separately and showed successful expression. The Au NW nanoinjector would find important biomedical applications in the fields such as gene therapy, DNA vaccination, targeted drug delivery, and probe/control of cell signaling events.

KEYWORDS: Au nanowire, nanoinjector, gene delivery, electrical trigger, DNA

$\mathrm{N}$ anomaterial-based devices interfacing with living cells have led to great progress in analysis and/or control of cellular behavior. Since one-dimensional nanomaterials can provide the best access into intracellular regions with minimum cellular damage, they are being widely used to study chemical and electrical characteristics of cells or deliver exogenous materials into cells. ${ }^{1-5}$ Precisely delivering biologically active molecules to a specific single cell can allow us to control fundamental cellular processes as well as provide us valuable clues to understanding them. Single cell transfection, for instance, can inject a specific gene or DNA into an embryonic stem cell to generate transgenic animals that show expression or knockout of a specific gene. ${ }^{6}$ This method can also deliver nucleic acids effectively into neurons or primary culture cells, which was rather difficult for global cell population transfection. ${ }^{7,8}$

It has been shown that quantum dots can be delivered to a single cell by using carbon nanotubes (CNTs) or BN nanotubes as nanoinjectors. ${ }^{9,10}$ Needles having nanoscale diameter can directly deliver desired biomaterials into a target cell while sustaining cell viability, not readily achievable by conventional techniques. ${ }^{11-14}$ Among the materials to be delivered into a cell, DNAs are particularly important. DNA delivery is a core fundamental technology for DNA vaccination and gene therapy and also useful for investigating gene functions and understanding underlying biological mechanisms. ${ }^{15,16}$ Recently, it has been reported that methods using a thin etched atomic force microscope (AFM) tip can deliver DNAs into a single cell, where an AFM tip attached by DNAs was inserted into the cell nucleus and DNAs were released by desorption. $^{17,18}$

An ideal physicochemical method for a direct delivery of bioactive materials into a cell should be able to implement precise timing, high spatial resolution, and quantitative molecular dosing while minimizing cell damage. We report here the direct gene delivery into the nucleus of a single living cell with high temporal and spatial selectivity using a newly developed $\mathrm{Au} \mathrm{NW}$ nanoinjector and successful expression of the delivered gene. A single-crystalline $\mathrm{Au}$ NW has the following superb characteristics that can make it an ideal intracellular DNA delivering device. First, the thinnest diameter among the DNA delivering devices as well as high flexibility and superstrength were achieved, which make it mechanically the best nanoneedle. ${ }^{19,20}$ Second, its atomically smooth and welldefined (111) surface makes it optimal for the attachment of DNAs via self-assembled monolayer (SAM). ${ }^{21,22}$ Third, its superb electric conductivity enables efficient electrochemical delivery of biomolecules into a cell. Finally, the highest spatial resolution and quantitative delivery were achieved, which are essential for well-controlled subcellular studies. Using the $\mathrm{Au}$

Received: January 27, 2013

Revised: April 19, 2013

Published: May 2, 2013 
NW injector, we were able to successfully deliver both a linear DNA fragment and a plasmid into the nucleus of a single cell and have observed successful expression of genes in both cases. Functional gene expression in the cell indicates that the Au NW injector intactly delivered DNAs into the cell without significantly affecting cell viability.

In addition to gene delivery, well-controlled electrotriggering pulses may allow the Au NW nanoinjector to deliver materials such as drugs or proteins to a cell at a specific phase of a cell cycle and cellular state, thereby controlling or monitoring the molecule-induced intracellular process with great timing precision.

Figure 1a schematically illustrates the Au NW nanoinjector system that delivers DNA molecules into a single cell using an electrical trigger. The movement of the $\mathrm{Au} \mathrm{NW}$ nanoinjector can be precisely controlled using a three-dimensional piezoelectric stage under optical monitoring. ${ }^{23,24}$ The property of $\mathrm{Au}$ that strongly scatters light makes it possible for a Au NW to be easily observed by an optical microscope. The Au NW injector can be accurately inserted into a specific intracellular position with high spatial resolution $(<200 \mathrm{~nm})$, limited by an optical microscope (Figure S1, Supporting Information). The experimental setup is comprised of a three-electrode system, a Au NW nanoinjector (working electrode), a saturated calomel electrode (reference electrode), and a Pt wire (counter electrode), allowing accurate application of an electric potential to the nanoinjector and efficient release of NW-attached DNA molecules into a cell.

We fabricated a $\mathrm{Au}$ NW nanoinjector by attaching a single $\mathrm{Au}$ NW to an adhesive-coated sharp tungsten (W) tip, and then insulating the tip with nail varnish (Figure S2, Supporting Information). The adhesive was silver-filled conductive paste (Norland Products Inc., NJ), which did not affect subsequent electrochemical reaction for releasing DNAs into a cell as adhesive was covered by insulating layer later. ${ }^{25}$ Figure $1 \mathrm{~b}$ shows a scanning electron microscope (SEM) image (i), an enlarged SEM image (ii), and an optical image (iii) of an asfabricated $\mathrm{Au}$ NW nanoinjector. The Au NWs were vertically grown on a c-cut sapphire substrate by the vapor transport method. ${ }^{26}$ The diameter and length of synthesized Au NWs are $136 \pm 30 \mathrm{~nm}$ and tens of micrometers, respectively, which were optimized by adjusting a synthetic parameter such as growth temperature in order to observe and manipulate them easily under an optical microscope. The Au NWs were attached on a tungsten tip leaving $20-30 \mu \mathrm{m}$ exposed. Au NWs are singlecrystalline with no defect and well-faceted by atomically smooth $\mathrm{Au}(111$ ) surfaces (Figure $\mathrm{lb}(\mathrm{ii})$ and Figure S3, Supporting Information).

Figure 1c shows an optical image of the nanoinjector penetrating into the nucleus of a cell. Importantly, a Au NW with a uniform diameter of 100-150 nm did not cause noticeable damage to the cell unlike tapered injectors. ${ }^{19}$ Furthermore, a $\mathrm{Au} \mathrm{NW}$ nanoinjector can act as an excellent nanoelectrode, allowing electrically triggered and thus rapid and quantitative delivery of DNA. While the delivery methods relying on the cleavage of disulfide bonds in the reducing environment of a cytoplasm require more than $15 \mathrm{~min}$ for dissociation reaction, ${ }^{9,10}$ a recently developed electrochemical approach, utilizing an electrical pulse, could readily deliver quantum dots attached on an electrode surface into a single cell in one minute. ${ }^{27}$ The $\mathrm{Au} \mathrm{NW}$ is highly competent at such electrochemical delivery. Since surface structures of the electrode critically influence electrochemical release, the well-
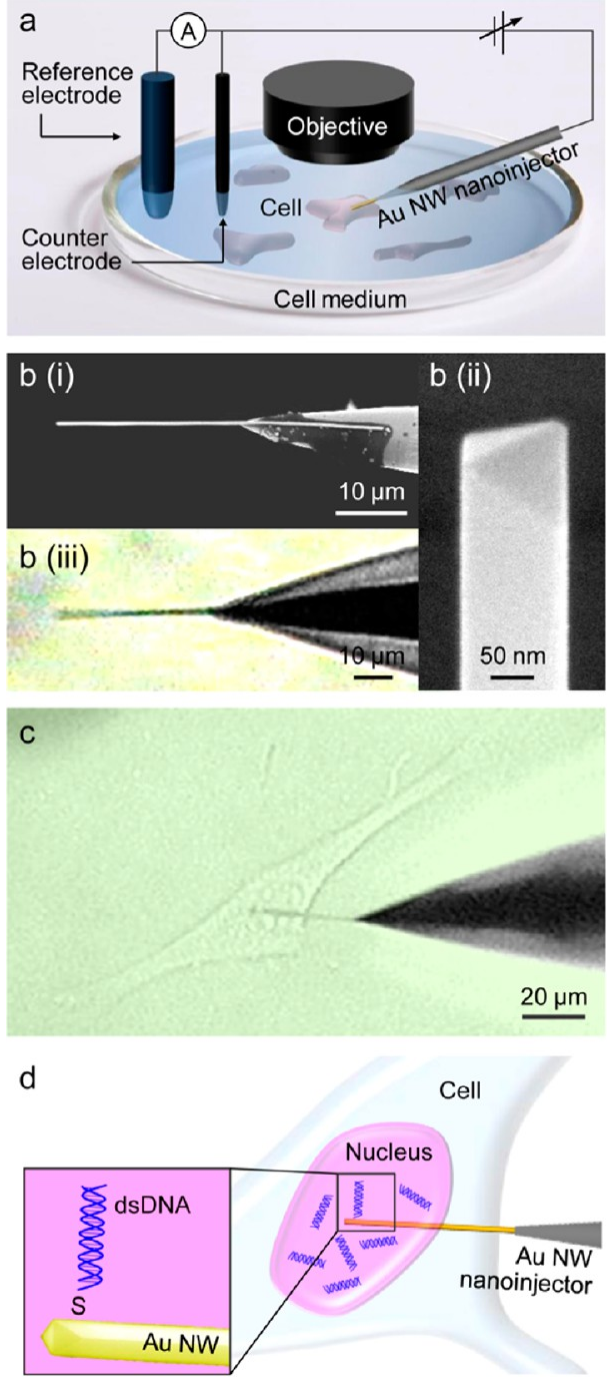

Figure 1. (a) Schematic illustration of a Au NW nanoinjector for electrically triggered delivery of gene into a single living cell. A DNAattached $\mathrm{Au} \mathrm{NW}$ nanoinjector is injected into a cell at designated position using a three-axis piezoelectric stage and then the DNA is released from a Au NW nanoinjector by applying an electric pulse. (b) SEM image before insulation (i), an enlarged SEM image of the tip (ii), and an optical image after insulation (iii) of an as-fabricated Au NW nanoinjector. (c) Optical image of a $\mathrm{Au}$ NW nanoinjector inserted into the nucleus of a living SK-N-SH cell. (d) Schematic illustration of DNA delivery into the nucleus of a living cell using a Au NW nanoinjector. The DNA is released from a Au NW by applying an electric pulse after DNA-attached Au NW nanoinjector is inserted into the nucleus.

defined and atomically smooth (111) surface of a Au NW produces more highly controlled reductive bond cleavage than surfaces not very well-defined. ${ }^{28,29}$ The electrochemical currents measured by the $\mathrm{Au} \mathrm{NW}$ electrode in a $50 \mathrm{mM}$ $\mathrm{H}_{2} \mathrm{SO}_{4}$ solution (Figure S4, Supporting Information) show oxidation and reduction peaks of a well-defined singlecrystalline $\mathrm{Au}(111)$ surface occurring at a constant potential with sharp peak shapes. ${ }^{30}$ All the DNAs on the NW could be directly delivered into the cell nucleus by applying an electric pulse for two minutes through the Au NW electrode (Figure 1d).

Figure $2 \mathrm{a}-\mathrm{c}$ shows that a $\mathrm{Au} \mathrm{NW}$ is highly flexible (also see Figure S5, Supporting Information). The Au NW could be even 


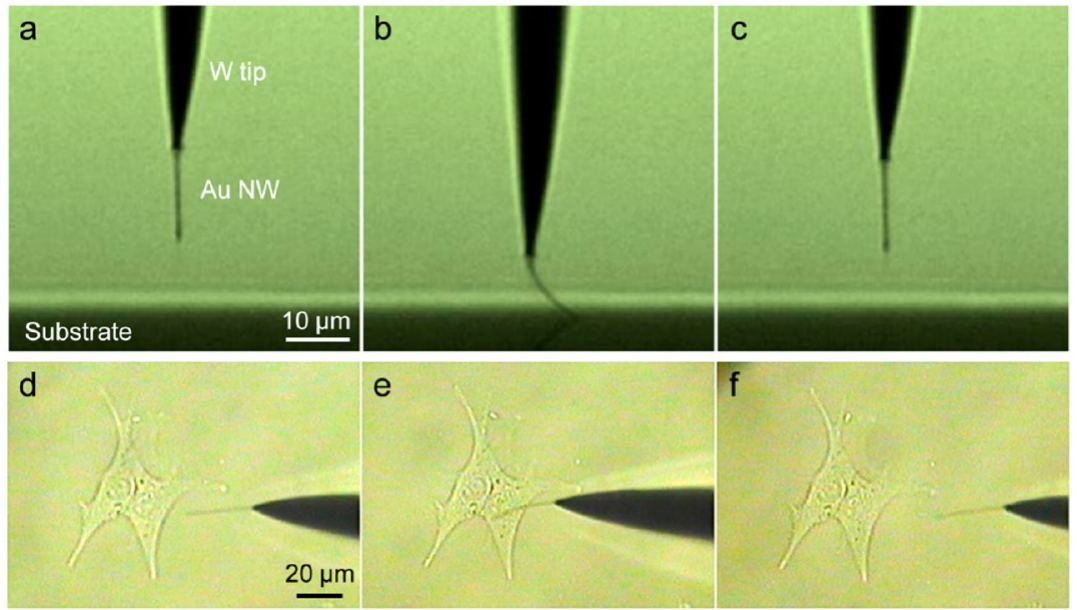

Figure 2. $(\mathrm{a}-\mathrm{c})$ Optical images showing flexible bending and complete recovery of a Au NW nanoinjector as the Au NW is pushed against a solid surface. (d-f) A Au NW nanoinjector was slightly bent on insertion into a cell. The NW recovered its initial straight shape on withdrawal. The NW was penetrating into the cell membrane at $\sim 5^{\circ}$ to the substrate plane to a depth of $15-20 \mu \mathrm{m}$ into the cytoplasm.

bent into a U-shape (Figure S6, Supporting Information) without breakage. On the other hand, Young's modulus for determining the stiffness of a $\mathrm{Au}$ NW is $72 \mathrm{GPa}$, which is larger than that for bulk $\mathrm{Au}(53-55 \mathrm{GPa})$ and very close to that of $\mathrm{Au}$ in a theoretical limit $(78 \mathrm{GPa}) .{ }^{31}$ This value suggests superstrength of a $\mathrm{Au}$ NW. The excellent flexibility and superstrength of a $\mathrm{Au}$ NW originates from its perfectly crystalline nature ${ }^{31}$ and can reduce cellular damage during the cell penetration. Figure $2 \mathrm{~d}-\mathrm{f}$ shows optical images taken when the $\mathrm{Au} N W$ was penetrating into the cell membrane at $\sim 5^{\circ}$ to the surface plane to the depth of $15-20 \mu \mathrm{m}$ into the cytoplasm. While the NW was slightly bent upon entering the cell (Figure $2 \mathrm{e})$, it immediately recovered its original straight shape upon withdrawal from the cell. These images suggest that upon cell penetration the flexible Au NW deflects around actin fibers that form a strong cytoskeleton and passes through the softer regions, thus minimizing cell deformation. ${ }^{19}$

In this study, we delivered both linear DNA and plasmid DNA into the cell, since DNAs are typically delivered into cells in these two types in biological and medical research. Usually plasmid is more efficiently delivered into the cell than linear DNA, because its circular double-stranded form is more stable in the cytoplasm and it can self-replicate. The delivery efficiency of linear DNA is considerably lower than that of plasmid because it is more readily degraded by intracellular nucleases. Introduction of linear DNA is necessary in some cases such as the generation of transgenic animals or investigation of viral genomes. We attached these two kinds of DNAs onto the $\mathrm{Au}$ NW utilizing different methods. The linear DNAs were attached to the $\mathrm{Au} \mathrm{NW}$ with a $\mathrm{Au}-\mathrm{S}$ bond after modifying the linear DNA by thiol at the 5 '-end. For plasmid attachment, we employed electrostatic attachment instead of using thiolated plasmid, of which synthesis is difficult and inefficient. The surface of a $\mathrm{Au} \mathrm{NW}$ was first attached by positively charged molecular species, and then it was attached by negatively charged plasmid using electrostatic attraction. ${ }^{32}$

In order to find the optimal potential of an electric pulse, the following experiments were performed. After immersing the $\mathrm{Au}$ NW nanoinejctor attached by the Cy3 dye-intercalated linear DNA in a buffer solution, electric potentials of $-0.7,-0.8$, and $-0.9 \mathrm{~V}$ were applied for $5 \mathrm{~min}$, respectively. The fluorescence intensities of the remaining $\mathrm{Cy} 3$ on the $\mathrm{NW}$ were measured after taking the nanoinjector out of the buffer solution. Most of fluorescence was eliminated by the electric pulse potential of -0.8 and $-0.9 \mathrm{~V}$ (Figure $3 \mathrm{a}$ and the blue bars in Figure 3d). ${ }^{33-35}$ To find an optimum duration of the potential, the fluorescence intensities were measured after applying a potential of -0.8 and $-0.9 \mathrm{~V}$ for $0,1,2$, and $3 \mathrm{~min}$, respectively. A potential of $-0.8 \mathrm{~V}$ for 2 min was found as an optimal potential and duration (Figure $3 \mathrm{a}$ and the magenta and green bars in Figure 3d). Similar optimization studies with living cells confirmed that an electrical trigger of the height of $-0.8 \mathrm{~V}$ and 2 min duration is most suitable for DNA delivery using the Au NW nanoinjector system. The same electric pulse condition was employed for releasing plasmid DNA.

Next, we examined if the Au NW nanoinjector can deliver a consistent amount of DNAs. We cultivated cells on four glass slides, selected $0,1,3$, or 6 cells per slide, and injected linear DNAs into each cell. Then, the amounts of delivered DNAs were measured by quantitative real-time polymerase chain reaction ( $\mathrm{qPCR}$ ) after extracting all DNAs from the cells cultured on a given slide. Figure 4 shows a plot of the amount of the delivered DNAs versus the total number of cells into which the target DNA had been injected. The linear proportionality indicates that the Au NW nanoinjector was able to deliver a consistent amount of DNAs into each cell.

It is vitally important to keep the cell alive when the bioactive materials are delivered into the cells. Thus, cell viability after $\mathrm{Au}$ NW nanoinjector-based DNA delivery was examined using trypan blue, which stains only the dead cells. We inserted a linear DNA-modified Au NW nanoinjector into a SK-N-SH cell grown on a gelatin-coated glass slide and applied electric pulses to a nanoinjector. Then, cell viability was examined by trypan blue staining. At $7 \mathrm{~h}$ after the injection, no intranuclear trypan blue staining was observed for all 13 cells examined. Furthermore, even after three times DNA delivery into the same cell at an interval of $5 \mathrm{~min}$, the cell was viable (no trypan blue staining; Figure S7, Supporting Information). These results suggest that repetitive intracellular DNA injection by the $\mathrm{Au} \mathrm{NW}$ nanoinjector does not cause apparent detrimental effect on cell viability.

Finally, we examined the successful expression of the delivered DNAs, which is important for studying cellular physiology after delivering desired DNA. Figure $5 \mathrm{a}-\mathrm{c}$ shows 

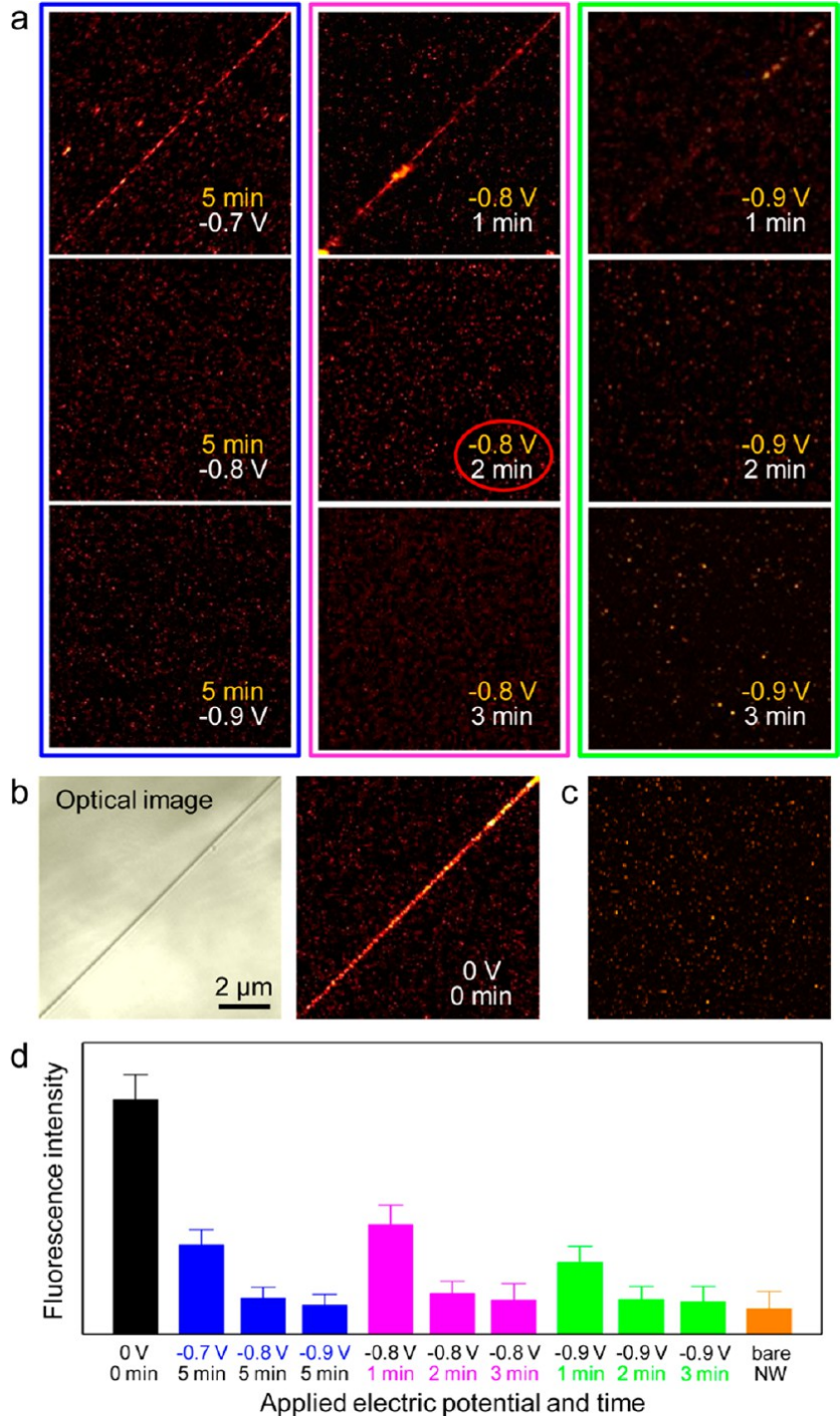

Figure 3. (a) Fluorescence images of Au NWs modified by Cy3incorporated DNA after applying electric pulses of different potential and duration. The potential and duration of applied electric pulse are written in the images. (b) Optical and fluorescence images of a $\mathrm{Au}$ NW before applying an electric pulse. (c) A fluorescence image of bare $\mathrm{Au}$ NW on which Cy3-labeled DNAs were not attached. (d) Plot of fluorescence intensity versus the potential and duration of applied electric pulses.

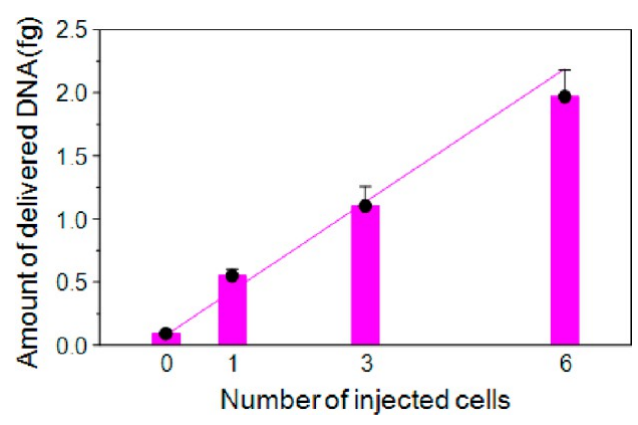

Figure 4. Plot of the amount of DNA delivered into cells versus the number of DNA-delivered cells by Au NW nanoinjectors. DNA was delivered into different number of cells (0, 1, 3, and 6). Electric potential of $-0.8 \mathrm{~V}$ was applied for $2 \mathrm{~min}$. Linear fit line shows that $\mathrm{Au}$ NW nanoinjectors can deliver DNA quantitatively.

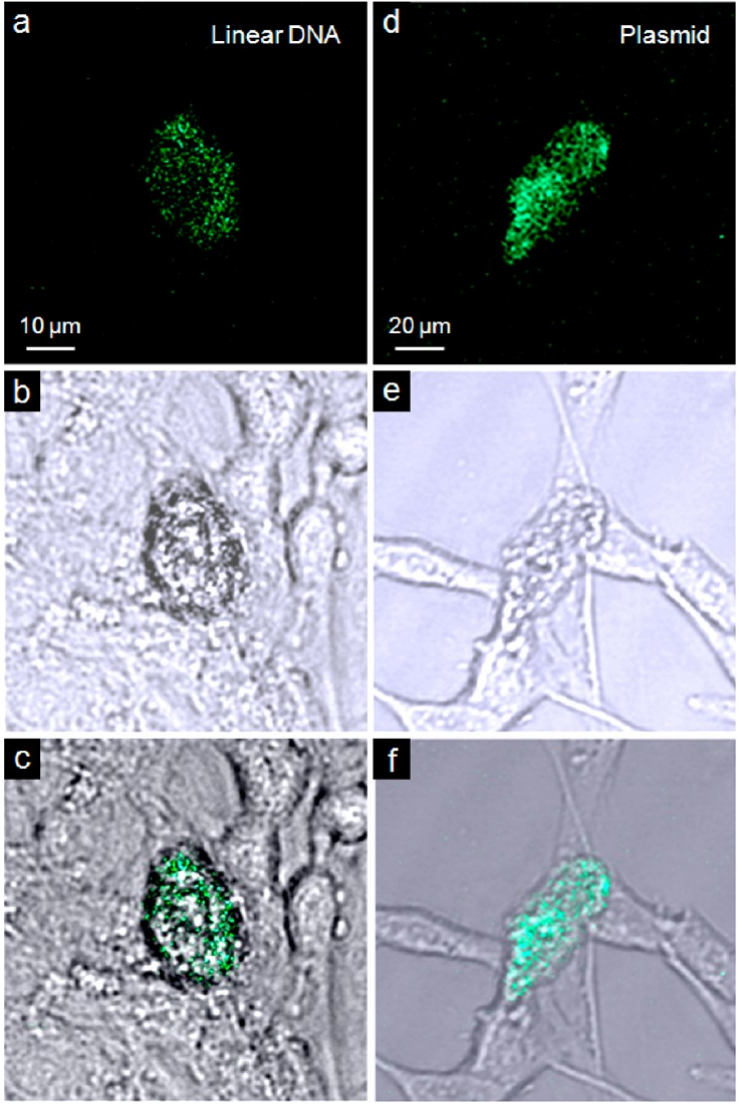

Figure 5. (a) Fluorescence, (b) optical, and (c) merged images of the SK-N-SH cell after delivery of linear DNA encoding GFP. The cell was grown in culture medium for $48 \mathrm{~h}$ after DNA delivery for GFP expression. (d) Fluorescence, (e) optical, and (f) merged image of the SK-N-SH cell after delivery of pEGFP-N1 encoding GFP. The cell was grown in culture medium for $48 \mathrm{~h}$ after plasmid delivery for GFP expression.

fluorescence, optical, and merged images, respectively, of cells grown in a culture medium for $48 \mathrm{~h}$ after delivering the thiolated linear DNA encoding green fluorescent protein (GFP). Distinct fluorescence signals were observed only from the cells that were injected with the linear DNA. Figure $5 \mathrm{~d}-\mathrm{f}$ show fluorescence, optical, and merged images, respectively, of the cells at $48 \mathrm{~h}$ after delivering pEGFP-N1, a plasmid containing the gfp gene. Similar to the case of linear DNA delivery, distinct fluorescence signals were observed only from the cells to which the plasmid was injected. When pEGFP-N1 plasmid DNA was released into cytoplasm by $\mathrm{Au}$ NW nanoinjectors, no distinct fluorescence was observed in any cells (Figure S8, Supporting Information). These results demonstrate that a Au NW nanoinjector can successfully deliver both linear and circular DNAs without damaging cells, driving efficient gene expression. The Au NW nanoinjector is able to maximize delivery efficiency of DNA by precise control of the location and amount of released DNA.

While DNAs attached to $\mathrm{Au} \mathrm{NW}$ nanoinjector can be released into cytoplasm as well as nucleus from the whole part of the $\mathrm{Au} \mathrm{NW}$, the resolution can be improved by further modification of the root part of a Au NW so that the DNAs are only attached to the NW tip part, although such modification was not necessary in this experiment. When genes are delivered into the nucleus via endocytosis and diffusion using liposomes or polymers as carriers, a very small portion $(\sim 0.3 \%)$ of the 
injected DNA reaches the nucleus due to DNA digestion and failure of nuclear membrane penetration. ${ }^{36}$ The quantitatively controlled DNA delivery can also minimize negative effects on a cell function or a cell cycle caused by excessive DNA remaining in the cytoplasm.

In conclusion, we report the development of a $\mathrm{Au} \mathrm{NW}$ nanoinjector for intracellular gene delivery to the nucleus of a single cell. The Au NW nanoinjector has several important features. First, it can deliver DNA directly into the nucleus without noticeable cellular damage. Second, electrical stimulation triggers the fast, quantitative, and timed delivery of DNA. Finally and most importantly, this system was able to deliver both linear DNA and plasmid DNA directly into the cell nucleus and allow successful gene expression. It is expected that the Au NW nanoinjector system would be widely used for the study of single cells to obtain more detailed information on gene functions and intracellular reactions, as well as for developing more advanced methods to deliver therapeutic DNA molecules for gene therapy and DNA vaccination.

\section{ASSOCIATED CONTENT}

\section{S Supporting Information}

Experimental details including supplementary figures and detailed descriptions of the fabrication and characterization of a $\mathrm{Au} \mathrm{NW}$ nanoinjector, preparation of DNA-attached NW nanoinjector, subcellular insertion of a NW nanoinjector, quantification of released target DNAs into cell, cell viability test, cell cultivation, and analytical instruments. This material is available free of charge via the Internet at http://pubs.acs.org.

\section{AUTHOR INFORMATION}

\section{Corresponding Author}

*E-mail: bongsoo@kaist.ac.kr (B.K.); leesy@kaist.ac.kr (S.Y.L).

\section{Author Contributions}

${ }^{\perp}$ S.M.Y. and M.K. contributed equally.

\section{Notes}

The authors declare no competing financial interest.

\section{ACKNOWLEDGMENTS}

The work of B.K. was supported by the Public Welfare and Safety research program through NRF-2012M3A2A1051686 and also by SRC 2012-0000648 funded by the Ministry of Science, ICT, and Future Planning. The work of S.Y.L. was supported by the Intelligent Synthetic Biology Center of Global Frontier Project funded by MEST (2011-0031963).

\section{REFERENCES}

(1) Duan, X.; Gao, R.; Xie, P.; Cohen-Karni, T.; Qing, Q.; Choe, H. S.; Tian, B.; Jiang, X.; Lieber, C. M. Nat. Nanotechnol. 2012, 7, 174179.

(2) Gao, R.; Strehle, S.; Tian, B.; Cohen-Karni, T.; Xie, P.; Duan, X.; Qing, Q.; Lieber, C. M. Nano Lett. 2012, 12, 3329-3333.

(3) Robinson, J. T.; Jorgolli, M.; Shalek, A. K.; Yoon, M.-H.; Gertner, R. S.; Park, H. Nat. Nanotechnol. 2012, 7, 180-184.

(4) Xie, C.; Lin, Z.; Hanson, L.; Cui, Y.; Cui, B. Nat. Nanotechnol. 2012, 7, 185-190.

(5) Shalek, A.; Robinson, J. T.; Karp, E. S.; Lee, J. S.; Ahn, D.-R.; Yoon, M.-H.; Sutton, A.; Jorgolli, M.; Gertner, R. S.; Gujral, T. S.; MacBeath, G.; Yang, E. G.; Park, H. Proc. Natl. Acad. Sci. U.S.A. 2010, 107, 1870-1875.

(6) DeChiara, T. M.; Poueymirou, W. T.; Auerbach, W.; Frendewey, D.; Yancopoulos, G. D.; Valenzuela, D. M. Methods Enzymol. 2010, 476, 285-294.

(7) Karra, D.; Dahm, R. J. Neurosci. 2010, 30, 6171-6177.
(8) Gresch, O.; Altrogge, L. Methods Mol. Biol. 2012, 801, 65-74.

(9) Chen, X.; Kis, A.; Zettl, A.; Bertozzi, C. R. Proc. Natl. Acad. Sci. U.S.A. 2007, 104, 8218-8222.

(10) Yum, K.; Na, S.; Xiang, Y.; Wang, N.; Yu, M.-F. Nano Lett. 2009, 9, 2193-2198.

(11) Aten, Q. T.; Jensen, B. D.; Tamowski, S.; Wilson, A. M.; Howell, L. L.; Burnett, S. H. Transgenic Res. 2012, 21, 1279-1290.

(12) Cai, D.; Mataraza, J. M.; Qin, Z.-H.; Huang, Z.; Huang, J.; Chiles, T. C.; Carnahan, D.; Kempa, K.; Ren, Z. Nat. Methods 2005, 2, 449-454.

(13) Yan, R.; Park, J.-H.; Choi, Y.; Heo, C.-J.; Yang, S.-M.; Lee, L. P.; Yang, P. Nat. Nanotechnol. 2012, 7, 191-196.

(14) Yum, K.; Yu, M. F.; Wang, N.; Xiang, Y. K. Biochim. Biophys. Acta 2011, 1810, 330-338.

(15) Alpar, H. O.; Papanicolaou, I.; Bramwell, V. W. Expert Opin. Drug Delivery 2005, 2, 829-842.

(16) Johnson-Saliba, M.; Jans, D. A. Curr. Drug Targets 2001, 2, 371399.

(17) Cuerrier, C. M.; Lebel, R.; Grandbois, M. Biochem. Biophys. Res. Commun. 2007, 355, 632-636.

(18) Han, S.-W.; Nakamura, C.; Kotobuki, N.; Obataya, I.; Ohgushi, H.; Nagamune, T.; Miyake, J. Nanomed.-Nanotechnol. Biol. Med. 2008 , $4,215-225$.

(19) Singhal, R.; Orynbayeva, Z.; Sundaram, R. V. K.; Niu, J. J.; Bhattacharyya, S.; Vitol, E. A.; Schrlau, M. G.; Papazoglou, E. S.; Friedman, G.; Gogotsi, Y. Nat. Nanotechnol. 2011, 6, 57-64.

(20) Tian, B.; Cohen-Karni, T.; Qing, Q.; Duan, X.; Xie, P.; Lieber, C. M. Science 2010, 329, 830-834.

(21) Love, J. C.; Estroff, L. A.; Kriebel, J. K.; Nuzzo, R. G.; Whitesides, G. M. Chem. Rev. 2005, 105, 1103-1169.

(22) Creager, S. E.; Hockett, L. A.; Rowe, G. K. Langmuir 1992, 8, $854-861$

(23) Yoon, I.; Kang, T.; Choi, W.; Kim, J.; Yoo, Y.; Joo, S.; Park, Q.H.; Ihee, H.; Kim, B. J. Am. Chem. Soc. 2009, 131, 758-762.

(24) Kang, T.; Yoo, S. M.; Yoon, I.; Lee, S. Y.; Kim, B. Nano Lett. 2010, 10, 1189-1193.

(25) Touwslager, F. J.; Gelinck, G.; Bouten, P. C. P. Improved Common Contact Layout for Flexible Displays. Eur. Pat. Appl. 2044652, 2009.

(26) Yoo, Y.; Seo, K.; Han, S.; Varadwaj, K. S. K.; Kim, H. Y.; Ryu, J. H.; Lee, H. M.; Ahn, J. P.; Ihee, H.; Kim, B. Nano Lett. 2010, 10, 432438.

(27) Yum, K.; Wang, N.; Yu, M.-F. Small 2010, 6, 2109-2113.

(28) Zhong, C.-J.; Zak, J.; Porter, M. D. J. Electroanal. Chem. 1997, $421,9-13$.

(29) Kawaguchi, T.; Yasuda, H.; Shimazu, K. Langmuir 2000, 16, 9830-9840.

(30) Kondo, T.; Morita, J.; Hanaoka, K.; Takakusagi, S.; Tamura, K.; Takahasi, M.; Mizuki, J.; Uosaki, K. J. Phys. Chem. C 2007, 111, 13197-13204.

(31) Seo, J. H.; Yoo, Y.; Park, N. Y.; Yoon, S. W.; Lee, H.; Han, S.; Lee, S. W.; Seong, T. Y.; Lee, S. C.; Lee, K. B.; Cha, P. R.; Park, H. S.; Kim, B.; Ahn, J. P. Nano Lett. 2011, 11, 3499-3502.

(32) Niidome, T.; Nakashima, K.; Takahashic, H.; Niidomec, Y. Chem. Commun. 2004, 17, 1978-1979.

(33) Huang, S.; Schopf, E.; Chen, Y. Nano Lett. 2007, 7, 3116-3121.

(34) Arinaga, K.; Rant, U.; Knezevic, J.; Pringsheim, E.; Tornow, M.; Fujita, S.; Abstreiter, G.; Yokoyama, N. Biosens. Bioelectron. 2007, 23, $326-331$.

(35) Takeishi, S.; Rant, U.; Fujiwara, T.; Buchholz, K.; Usuki, T.; Arinaga, K.; Takemoto, K.; Yamaguchi, Y.; Tornow, M.; Fujita, S.; Abstreiter, G.; Yokoyama, N. J. Chem. Phys. 2004, 120, 5501-5504.

(36) Luo, D.; Saltzman, W. M. Nat. Biotechnol. 2000, 18, 33-37. 